\title{
Tendência temporal do tétano acidental no período de 1981 a 2004 em Pernambuco com avaliação do impacto da assistência em unidade de terapia intensiva sobre a letalidade
}

\author{
Mortality trend due to accidental tetanus from 1981 to 2004 in \\ Pernambuco and analysis of the impact on intensive care unit attendance
}

\author{
Pedro Alves da Cruz Gouveia', Cláudia Elise Ferraz Silva², \\ Demócrito de Barros Miranda Filho ${ }^{3}$, Sylvia Nery Bernardino ${ }^{2}$, \\ Abelardo Gonçalves Escarião ${ }^{2}$ e Ricardo Arraes de Alencar Ximenes ${ }^{4}$
}

\begin{abstract}
RESUMO
Apesar da redução na incidência de tétano acidental no Brasil, não houve queda significativa na letalidade. Nesta série de casos, comparamos a letalidade antes e após o estabelecimento padrão de manejo em unidade de terapia intensiva do paciente com tétano no Hospital Universitário Oswaldo Cruz, no período de 1981 a 2004. Em 24 anos, foram internados 1.971 pacientes e antes do manejo em Unidade de terapia intensiva a letalidade era de 35\%. Durante 1997 foi instituída a unidade de terapia intensiva para assistência dos pacientes com tétano, e de 1998 a 2004, a letalidade caiu para $12,6 \%, \mathrm{OR}=0,27$ (IC95\%= 0,18- 0,39); p <0,001. Esta tendência foi evidenciada em todas as faixas etárias e em ambos os sexos. A centralização da assistência a esses pacientes em um único serviço especializado com Unidade de terapia intensiva de forma precoce, portanto, tem sido decisiva na redução da letalidade, por contar com a vasta experiência da equipe de saúde no manejo do tétano e melhor tratamento sintomático, antecipando as graves complicações da doença.
\end{abstract}

Palavras-chaves: Tétano acidental. Letalidade. Unidade de terapia intensiva.

\begin{abstract}
Despite reductions in the incidence of accidental tetanus cases in Brazil, there has not been any significant decrease in its mortality. In this case series, the mortality rates before and after establishing standard management practices for tetanus patients in the intensive care unit at the 0swaldo Cruz University Hospital are compared over the period from 1981 to 2004. Over these 24 years, 1.971 patients were admitted. Before establishing the intensive care unit management, the mortality rate was 35\%. The Intensive care unit for attending to tetanus patients was established in 1997 . From 1998 to 2004 , the mortality rate fell to $12.6 \%$ : $\mathrm{OR}=0.27(95 \% \mathrm{CI}=0.18-0.39) ; \mathrm{p}<0.001$. This trend was seen in all age groups and both sexes. The centralization of attendance for these patients into a single specialized service with early treatment in an intensive care unit has therefore been decisive in reducing the mortality rate. This service can count on the medical team's vast experience of tetanus management, with better treatment of symptoms that forestalls the serious complications from this disease.
\end{abstract}

Key-words: Accidental tetanus. Mortality. Intensive care unit.

O tétano acidental ainda é um grave problema de saúde pública, apesar de mais de um século da descoberta da vacina antitetânica. Em países subdesenvolvidos, é responsável por cerca de 500.000 óbitos/ano ${ }^{21}$. Nos países desenvolvidos, onde a doença passou a ser relativamente rara, a maioria dos casos notificados ocorre entre os indivíduos idosos ${ }^{16}$.

\footnotetext{
1. Hospital das Clínicas, Universidade Federal de Pernambuco, Recife-PE. 2. Hospital Universitário Oswaldo Cruz, Recife, PE. 3. Departamento de Medicina Clínica, Faculdade de Ciências Médicas, Universidade de Pernambuco, Recife, PE. 4. Departamento de Medicina Tropical, Centro de Ciências da Saúde, Universidade de Pernambuco, Recife, PE.

Endereço para correspondência: Dr. Pedro Alves da Cruz Gouveia. Rua Dom Sebastião Leme 57/301, 52011-160 Recife, PE.

Tel: $55818884-8464$.

e-mail: pedroalves@click21.com.br

Recebido para publicação em 23/09/2008

Aceito em 19/01/2009
}

Estima-se em aproximadamente um milhão, o número de casos/ano de tétano no mundo, com taxa de letalidade variando de 10 a $53 \%{ }^{412} 1819$.

No Brasil, o coeficiente de incidência por 100.000 habitantes caiu de 1,8 na década de 1980 para 0,32 em 2000, talvez pela maior eficiência das campanhas de vacinação principalmente em menores e gestantes ${ }^{520}$. Apesar da queda da incidência, não há um avanço significativo quanto à diminuição da letalidade, que permanece em torno de $30 \% 5$.

A alta letalidade da doença é decorrente de sua gravidade clínica e de suas complicações. 0 tratamento sintomático atual envolve suporte em unidade de terapia intensiva (UTI) com sedação intensa, bloqueio neuromuscular e assistência ventilatória mecânica por semanas nos casos de tétano moderado a gravíssimo ${ }^{117}$. Mesmo nos melhores centros, a letalidade ainda 
é alta, talvez devido à doença ou a complicações associadas à terapia intensiva ${ }^{1}$. Brauner e cols ${ }^{2}$ demonstraram decréscimo da mortalidade pelo tétano, após melhorias nos cuidados de UTI e tratamento da instabilidade autonômica, caindo de 36,5\% para $18 \%^{2}$.

A região Nordeste concentra um pouco mais de um terço dos casos de tétano do País 5 . Em 2004, 15\% dos casos notificados na região foram do Estado de Pernambuco. Quarenta casos de tétano acidental por ano têm sido notificados, em média, no estado nos últimos seis anos ${ }^{11}$.

Em Pernambuco, o Hospital Universitário Oswaldo Cruz é a única unidade de referência no tratamento de pacientes com tétano. Desde 1997, esta instituição dispõe de unidade de terapia intensiva para doenças infecciosas (UTI - DIP).

Este estudo propõe-se a descrever o impacto da UTI sobre a letalidade por tétano e a tendência da doença quanto ao número de casos e letalidade em Pernambuco, no período de 1981 a 2004.

\section{MATERIAL E MÉTODOS}

Trata-se de estudo descritivo retrospectivo, tipo série de casos, com dois tipos de análise: avaliação da tendência temporal da letalidade e do número de casos de tétano no Estado de Pernambuco; estudo de caráter analítico com comparação da letalidade entre dois grupos: antes e após a UTI.

Para comparação de grupos, foram consideradas as variáveis sexo e idade, categorizada em três faixas etárias: 12 a 30 anos, 31 a 50 e maiores de 50 anos.

0 estudo foi realizado no Hospital Universitário Oswaldo Cruz, Recife, PE, no período de 1981 a 2004. O Hospital-Escola é referência regional em doenças infecciosas e parasitárias e está inserido no Sistema Único de Saúde (SUS), sendo o único centro de referência para tratamento de tétano no estado. 0 intervalo de tempo estudado envolve os períodos antes e após o estabelecimento da UTI-DIP, respectivamente, de 1981 a 1996 e de 1997 a 2004.

A coleta de dados foi realizada em formulário confeccionado especificamente para este estudo, utilizando-se informações de prontuários médicos, dos pacientes maiores de 12 anos de idade com diagnóstico de tétano acidental, obtidos do arquivo médico do hospital. Para construção do banco de dados e análise, foi utilizado o programa Epiinfo versão 6.04. As informações foram digitadas em dupla entrada. Ao final da digitação, os dois bancos de dados foram comparados e, a seguir, eventuais erros e discordâncias foram corrigidos, após nova consulta aos formulários.

\section{RESULTADOS}

Em um período de 24 anos, foram internados no HUOC 1.971 pacientes com diagnóstico de tétano. Foram excluídos dois casos, por perda de seguimento (uma evasão e uma transferência). Observou-se predomínio do sexo masculino na proporção de
4:1. A média de letalidade, em todo o período, foi de $31,8 \%$ (Tabela 1).

A letalidade foi maior entre os maiores de 50 anos (44,6\%), em comparação com as faixas etárias mais jovens: 12 a 30 anos $(24,1 \%)$ e 31 a 50 anos $(28,4 \%)$ e esta diferença foi estatisticamente significante ( $\mathrm{p}<0,001 \mathrm{em}$ ambos).

Constatou-se diferença na taxa de letalidade entre homens e mulheres: $27,9 \%$ e 46,9\%, respectivamente (Tabela 1). Após estratificação, por faixa etária, a diferença foi estatisticamente significante entre os menores de 30 anos e os maiores de 50 anos.

Observou-se tendência decrescente do número de casos de tétano acidental, nos últimos 20 anos. A média de casos de tétano por ano na década de oitenta foi de 117,2 caindo para 35 casos/ano após o ano 2000 (Figura 1).

Entre 1981 e 1996, o percentual de óbitos foi de 35\%. Durante o ano de 1997, foi instituída a UTI para a assistência dos pacientes com tétano. A partir de 1998, a taxa de letalidade caiu para 12,6\% (Figura 1). A queda de letalidade foi observada em ambos os sexos e em todas as faixas etárias com significância estatística (Tabela 2).

\section{TABELA 1}

Letalidade de tétano por sexo e faixa etária.

\begin{tabular}{lrrc}
\hline & \multicolumn{2}{c}{ Pacientes } & Letalidade \\
\cline { 2 - 3 } & \multicolumn{1}{c}{$\mathrm{n}^{\mathrm{o}}$} & $\%$ & $\%$ \\
\hline Sexo & 1.568 & 78,6 & \\
$\quad$ masculino & 401 & 20,4 & 27,9 \\
feminino & & & 46,9 \\
Faixa etária (anos) & 780 & 39,6 & \\
$12-30$ & 570 & 28,9 & 24,1 \\
$31-50$ & 619 & 31,5 & 28,4 \\
$>50$ & 1.969 & 100,0 & 44,6 \\
\hline Total & & & 31,8 \\
\hline
\end{tabular}

TABELA 2

Letalidade pré e pós UTI estratificado por faixa etária e sexo e associação entre manejo em UTI e letalidade*.

\begin{tabular}{|c|c|c|c|c|c|c|}
\hline & \multicolumn{2}{|c|}{$\begin{array}{c}\text { Pré-UTI } \\
(1981-1996)\end{array}$} & \multicolumn{2}{|c|}{$\begin{array}{c}\text { Pós-UTI } \\
(1998-2004)\end{array}$} & \multirow{3}{*}{$\begin{array}{c}\text { Odds-Ratio } \\
\text { (IC 95\%) }\end{array}$} & \multirow{3}{*}{$\begin{array}{l}\text { Valor } \\
\text { de "p" }\end{array}$} \\
\hline & casos & letalidade & casos & letalidade & & \\
\hline & $\left(\mathrm{n}^{0}\right)$ & $(\%)$ & $\left(\mathrm{n}^{\mathrm{o}}\right)$ & (\%) & & \\
\hline $12-30$ anos & 699 & 25,9 & 60 & 5,0 & $0,15(0,05-0,45)$ & $<0,001$ \\
\hline masculino & 611 & 24,5 & 52 & 3,8 & $0,12(0,03-051)$ & $<0,001$ \\
\hline feminino & 88 & 35,2 & 8 & 12,5 & $0,26(0,03-2,23)$ & 0,36 \\
\hline $31-50$ anos & 443 & 33,6 & 115 & 10,4 & $0,23(0,12-0,43)$ & $<0,001$ \\
\hline masculino & 372 & 33,6 & 107 & 10,3 & $0,23(0,12-0,44)$ & $<0,001$ \\
\hline feminino & 71 & 33,8 & 8 & 12,5 & $0,28(0,03-2,41)$ & 0,40 \\
\hline$>50$ anos & 489 & 49,3 & 103 & 19,4 & $0,25(0,15-0,42)$ & $<0,001$ \\
\hline masculino & 301 & 41,5 & 79 & 13,9 & $0,23(0,12-0,45)$ & $<0,001$ \\
\hline feminino & 188 & 61,7 & 24 & 37,2 & $0,37(0,15-0,90)$ & 0,04 \\
\hline Total & 1631 & 35,0 & 378 & 12,6 & $0,27(0,18-0,39)$ & $<0,001$ \\
\hline
\end{tabular}

*excluído o ano de 1997, quando a UTI estava em consolidação.

UTI: unidade de terapia intensiva, IC 95\%: intervalo de confiança 95\% 


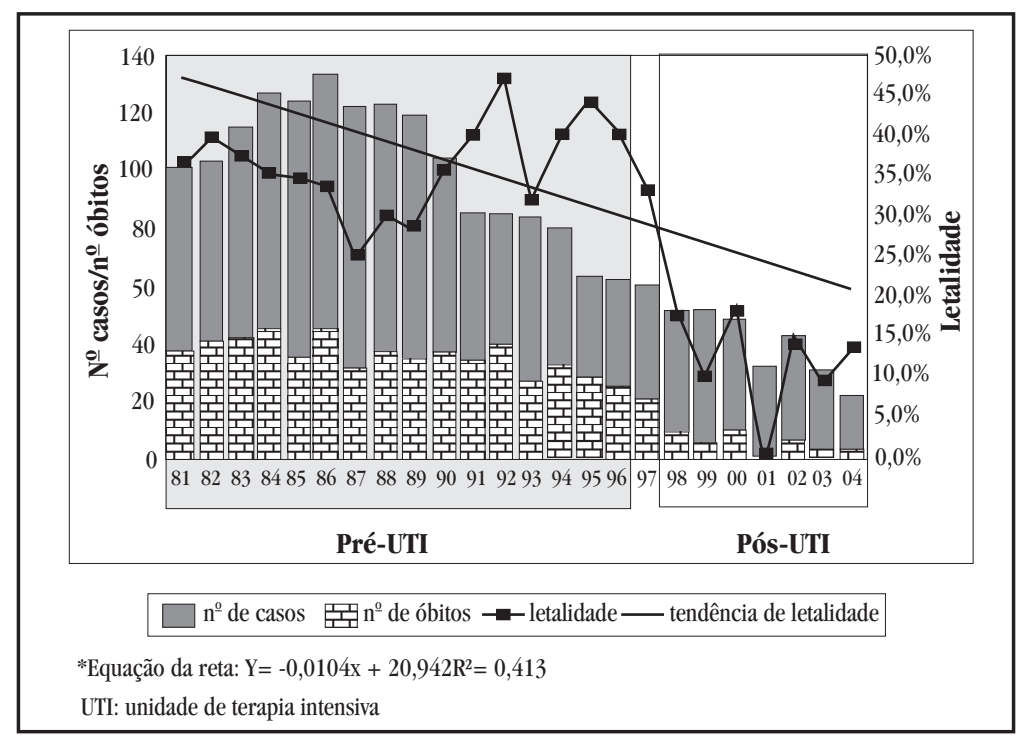

FIGURA 1

Tendência temporal de casos, óbitos e letalidade por tétano.

\section{DISCUSSÃo}

Este estudo descreve o comportamento epidemiológico do tétano em Pernambuco, em 24 anos, e envolve quase dois mil pacientes tratados em um só serviço de referência que concentra 0 atendimento de mais de $90 \%$ dos casos do estado. Observou-se uma tendência de queda no número de casos de tétano acidental em ambos os sexos ao longo do período. A taxa de letalidade, no entanto, só caiu a partir de 1998 (35\% para 12,6\%), após a instituição da UTI para a assistência dos pacientes com tétano. A letalidade foi maior entre os idosos e nas mulheres.

A queda progressiva do número de casos de tétano observada no período estudado está em consonância com os dados do Ministério da Saúde do Brasil. Melhor cobertura vacinal e assistência a feridas em caso de trauma, assim como ganhos na educação em saúde da população parecem ter participação neste fato ${ }^{2022}$.

0 predomínio do sexo masculino $(79,6 \%)$, em todas as idades, é descrito em estudos brasileiros e de outros países. No Brasil, as mulheres grávidas são vacinadas no pré-natal; além disso, os indivíduos do sexo masculino costumam ser mais expostos a ferimentos ${ }^{2822} 23$.

0 acometimento de indivíduos mais idosos é uma tendência já constatada em países desenvolvidos e em alguns Estados do Brasil, como São Paulo e Rio Grande do Sul, reforçando a necessidade de incremento da imunização nesta faixa etária ${ }^{78}{ }^{1015}$. Os níveis protetores de anticorpos para o tétano decrescem com o avançar da idade ${ }^{1524}$. Weckx e cols demonstraram em trabalho recente, através da análise do nível sérico da antitoxina, que 94\% dos idosos eram suscetíveis ao tétano, e que uma única dose do toxóide tetânico não era capaz de imunizar adequadamente este grupo ${ }^{24}$. Uma série completa de vacinação antitetânica parece, portanto, ser necessária para a prevenção do tétano nessa faixa etária.
A maior letalidade em indivíduos acima de 50 anos, descrita neste estudo, é similar a de outras séries ${ }^{6}{ }^{14}$. Outros autores descrevem idade avançada como um fator de risco independente para morte por tétano ${ }^{1821}$.

Nesta série, a letalidade entre as mulheres foi mais alta que a dos homens, principalmente na faixa etária abaixo de 30 e acima de 50 anos. Alguns estudos realizados para identificar fatores preditivos de morte descrevem maior letalidade entre mulheres, com diferença estatisticamente significante na análise univariada. No entanto, quando é feito um ajuste para outros fatores em análise multivariada, a variável sexo não permanece no modelo final da regressão logística ${ }^{13} 1821$.

Neste estudo, observou-se uma tendência de redução da letalidade pelo tétano no decorrer de mais de duas décadas, em Pernambuco. A taxa de letalidade na década de 1980, em torno de 35\%, foi similar à média brasileira e do Nordeste do país 5 . No entanto, após o final da década de 1990, com a introdução dos cuidados de terapia intensiva, a letalidade em Pernambuco caiu para 12,6\%, enquanto permaneceu estável na região Nordeste e no país como um todo. A redução da letalidade após manejo em UTI teve significância estatística $(\mathrm{p}<0,001)$ e foi observada em ambos os sexos e em todas as faixas etárias.

0 Estado de Pernambuco tem sido pioneiro em atuar de forma centralizada no atendimento e hospitalização de pacientes com tétano. A assistência a estes pacientes ocorre no Hospital Universitário Oswaldo Cruz, para o qual são referidos quase $100 \%$ dos casos. Conseqüentemente, a letalidade descrita neste estudo expressa a letalidade por tétano em todo o estado, sendo, portanto similar aos dados oficiais do Ministério da Saúde. A atual taxa de letalidade observada neste trabalho é comparável àquelas encontradas em países desenvolvidos (10-17\% $)^{31221}$.

Em contraponto, as taxas de letalidade descritas em outros estados do Brasil ainda são muito altas distanciando-se daquela observada em Pernambuco. Estados como São Paulo, 
Rio de Janeiro e Rio Grande do Sul apresentam taxas de letalidade por tétano em torno de 30-42\%. Em Santa Catarina observouse queda na incidência da doença, mas não na letalidade, que permanece em torno de $42 \%{ }^{23}$. Esses estados possuem rede de serviços de saúde complexa, com hospitais de referência com elevado padrão de assistência e de tecnologia médica. 0 fato de não atingirem patamares tão baixos de letalidade quanto os do Estado de Pernambuco deve-se fundamentalmente a dois fatores. 0 primeiro, corresponde à centralização da assistência ao paciente com tétano, permitindo que 0 atendimento seja realizado por uma equipe qualificada e treinada, e que vem acumulando uma vasta experiência ao longo do tempo. Como o tratamento desses pacientes, requer cuidados e procedimentos específicos, a experiência da equipe que presta atendimento aos mesmos pode ter uma influência decisiva no desfecho da doença. 0 segundo, é que essa centralização ocorre em uma UTI especializada onde são internados todos os pacientes com tétano. 0 impacto do atendimento, a esse tipo de paciente em UTI, é bem conhecido e pode ser ilustrado pela experiência de dois hospitais em Porto Alegre, nos quais se observou uma queda da letalidade por tétano de $36,5 \%$, no período anterior à introdução dos cuidados de em terapia intensiva, para $18 \%$, no período posterior à introdução dos mesmos ${ }^{2}$. E como nosso estado, há uma normatização da assistência ao tétano em UTI específica precocemente, há um melhor tratamento sintomático, antecipando as graves complicações da doença, com miorrelaxantes, sedativos, bloqueio neuromuscular e assistência ventilatória mecânica.

A despeito da importante redução da ocorrência de casos de tétano, as taxas de letalidade no Brasil persistem acima de $30 \%$, demonstrando a necessidade de melhor planejamento e qualidade na assistência a este tipo de paciente ${ }^{25}$. A experiência de Pernambuco aponta que a centralização da assistência a esse paciente em serviço especializado, com unidade de terapia intensiva, tem sido decisiva na redução da letalidade. Essa estratégia deve ser considerada quando da (re)definição de modelos assistenciais a pacientes com tétano.

\section{REFERÊNCIAS}

1. Attygalle D, Rodrigo N. New trends in the management of tetanus. Expert Review of Anti-Infective Therapy 2: 73-84, 2004.

2. Brauner JS, Vieira SR, Bleck TP.Changes in severe accidental tetanus mortality in the ICU during two decades in Brazil. Intensive Care Medicine 28: 930-935, 2002.

3. Centers of Disease Control and Prevention. Tetanus Surveillance - United States, 1995-1997. CDC surveillance summaries: Morbidity and Mortality Weekly Report 47: 1-13, 1998.

4. Edmondson RS, Flowers MW. Intensive care in tetanus: management, complications, and mortality in 100 cases. British Medical Journal 1: 1401-1404, 1979 .
5. Fundação Nacional de Saúde. Boletim Epidemiológico: Evolução temporal das doenças de notificação compulsória no Brasil de 1980 a 1998. Centro Nacional de Epidemiologia, Ministério da Saúde, Brasília 1999.

6. Greco Jiuseppe B, Tavares-Neto J, Greco Jr JB. Accidental tetanus: prognosis evalution in a historical series at a hospital in Salvador, Bahia, Brazil. Revista do Instituto de Medicina Tropical de São Paulo 45: 35-40, 2003.

7. Hainz U, Jenewein B, Asch E, Pfeiffer KP, Berger P, Grubeck-Loebenstein B. Insufficient protection for healthy elderly adults by tetanus and TBE vaccines. Vaccine 23: 3232-3235, 2005.

8. Lima VMSF, Garcia MT, Resende MR, Nouer AS, Campos EOM, Papaiordanou PMO, Silva LJ. Tétano acidental: análise do perfil clínico e epidemiológico de casos internados em hospital universitário. Revista de saúde pública 32: 166-171, 1998.

9. Litvoc J, Leite RM, Katz G. Aspectos epidemiológicos do tétano no Estado de São Paulo (Brasil). Revista do Instituto de Medicina Tropical de São Paulo 33: 477-484, 1991.

10. Mayol CV, Sanza AIC, Ortega AE, Caso JAG. El reto de la protección antitetánica en los ancianos. Atencion Primaria/Sociedad Espanola de Medicina de Família y Comunitaria 33: 524-525, 2004.

11. Ministério da Saúde/ Sinan/ Secretaria de Vigilância em Saúde, Brasília 2007. http://portal.saude.gov.br/portal/arquivos/pdf/casos_tetano_acidental.pdf. Acessado em 07/07/2008.

12. Miranda-Filho DB, Ximenes RA, Barone AA, Vaz VL, Vieira AG, Albuquerque VM. Randomised controlled trial of tetanus treatment with antitetanus immunoglobulin by the intrathecal or intramuscular route. British Medical Journal 328: 615-617, 2004.

13. Miranda-Filho DB, Ximenes RA, Bernardino SN, Escarião AG. Identification of risk factors for death from tetanus in Pernambuco, Brazil: a case-control study. Revista do Instituto de Medicina Tropical de São Paulo 42: 333-339, 2000.

14. Moraes EN, Pedroso ER. Tétano no Brasil: doença do idoso? Revista da Sociedade Brasileira de Medicina Tropical 33: 271-275, 2000.

15. Oztürk A, Göahmetoglu S, Erdem F, Mýsgüroglu Alkan S. Tetanus antitoxin levels among adults over 40 years of age in Central Anatolia, Turkey. Clinical Microbiology and Infection 9: 33-38, 2003.

16. Pedalino B, Cotter B, Ciofi degli Atti M, Mandolini D, Parrocini S, Salmaso S. Epidemiology of tetanus in Italy in years 1971-2000. Euro Surveillance 7: 103-110, 2002.

17. Rodrigues Júnior GR, Amaral JLG. Experiência clínica com o uso de sedativos em terapia intensiva: estudo retrospectivo. Revista Brasileira de Anestesiologia 52: 747-755, 2002.

18. Saltoglu N, Tasova Y, Midikli D, Borgut R, Dündar IH. Prognostic factors affecting deaths from adult tetanus. Clinical Microbiology and Infection 10: 229-233, 2004.

19. Sanford JP. Tetanus - Forgotten but not gone. New England Journal of Medicine 332: 812-813, 1995.

20. Secretaria de Vigilância em Saúde. Guia de vigilância epidemiológica $6^{\circ}$ edição. Ministério da Saúde, Brasília 2005.

21. Thwaites CL, Yen LM, Glover C, Tuan PQ, Nga NTN, ParryJ, Loan HT, Bethel D, Day NP, White NJ, Soni N, Farrar JJ. Predicting the clinical outcime of tetanus: the tetanus severity score. Tropical Medicine and International Health 11: 279-287, 2006.

22. Veronesi R, Focaccia R, Tavares W, Mazza CC. Tétano. In: Veronesi (ed) Tratado de infectologia, $3^{\text {a }}$ edição, Editora Atheneu, São Paulo, p. 1115-1138, 2005.

23. Viertel IL, Amorim L, Piazza U. Tétano acidental no Estado de Santa Catarina. Brasil: aspectos epidemiológicos. Epidemiologia e Serviços de Saúde 14: 33-40, 2005.

24. Weckx LY, Divino-Goes K, Lihama DM, Carraro E, Bellei N, Granato CF, MoraesPinto MI. Effect of a single tetanus-diphtheria vaccine dose on the immunity of elderly people in São Paulo, Brazil. Brazilian Journal of Medical and Biological Research 39: 519-523, 2006. 